\title{
A Brief Communication on the Improved Mechanism of Graphene on Zinc-Rich Coatings
}

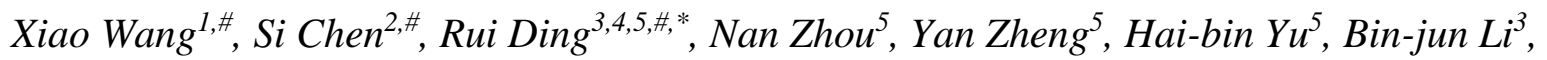 \\ Tai-jiang Gui ${ }^{1}$, Wei-hua $\mathrm{Li}^{2,4}$,* \\ ${ }^{1}$ Marine Chemical Research Institute, State Key Laboratory of Marine Coatings, Qingdao 266071, \\ China; \\ ${ }^{2}$ College of Chemical Engineering and Technology, Sun Yat-sen University, Zhuhai, 519082, China; \\ ${ }^{3}$ College of Oceanology, Yantai University, Yantai, 264005, China; \\ ${ }^{4}$ Key Laboratory of Marine Environmental Corrosion and Bio-fouling, Institute of Oceanology, \\ Chinese Academy of Science, Qingdao 266071, China; \\ ${ }^{5}$ Key Laboratory of Marine Materials and Related Technologies, Zhejiang Key Laboratory of Marine \\ Materials and Protective Technologies, Ningbo Institute of Materials Technologies and Engineering, \\ Chinese Academy of Sciences, Ningbo 315201, China; \\ \# These authors contributed equally to this work. \\ *E-mail: dmoyuyang@foxmail.com (Rui Ding); liweihua@qdio.ac.cn (Wei-hua Li)
}

doi: $10.20964 / 2019.04 .26$

Received: 9 December 2018 / Accepted: 18 January 2019 / Published: 10 March 2019

Gallic acid-based epoxy resin monomer was synthesized by the natural extract of gallnut for the efficient dispersion of graphene in epoxy resin. The graphene zinc-rich anticorrosion coatings were further prepared on the basis of the graphene / gallic acid-based epoxy resin monomer dispersion system. Electrochemical experiments and spectral characterization were carried out to study the effect of graphene on zinc-rich coatings and its mechanism. Experiments showed that graphene enhanced the cathodic protection currents of the zinc-rich coatings and prolonged the cathodic protection time. Simultaneously, graphene slowed down the penetration of corrosive media into the coatings. The conductivity of graphene improved electrical contact between zinc particles as well as zinc and iron, and transformed un-activated zinc particles into activated zinc particles. The dispersed and layered structure of the graphene extended the permeation path of the corrosive medium, which resulted in reduced permeation rate and decreased water content of the coatings. It was demonstrated by the tracking of zinc corrosion products by micro-area Raman spectroscopy.

Keywords: Corrosion, Graphene, Zinc-rich coatings, Electrochemistry

\section{$\underline{\text { FULL TEXT }}$}


(C) 2019 The Authors. Published by ESG (www.electrochemsci.org). This article is an open access article distributed under the terms and conditions of the Creative Commons Attribution license (http://creativecommons.org/licenses/by/4.0/). 